\title{
LAMELLAR KERATOPLASTY IN THE MANAGEMENT OF INFLAMMATORY CORNEAL ULCERATION AND PERFORATION
}

\author{
D. A. R. BESSANT and J. K. G. DART \\ London
}

\begin{abstract}
SUMMARY
Corneal ulceration and perforation may occur in the course of several systemic and ocular inflammatory conditions. These serious complications may respond to systemic immunosuppression and conjunctival surgery, but tectonic keratoplasty is sometimes required. We report on 10 cases of corneal ulceration (of which 8 suffered perforation) in patients with rheumatoid arthritis, Stevens-Johnson syndrome and Mooren's ulceration which were treated by lamellar keratoplasty. Our microsurgical technique for lamellar keratoplasty benefited from the use of viscoelastic agents, cyanoacrylate adhesive and an adjustable stepped diamond knife. These recent advances have made this procedure as easy to perform as a penetrating keratoplasty. Lamellar keratoplasty virtually eliminates the risk of graft rejection and reduces the risk of perforation in the event of a subsequent exacerbation of the melting process. Surgery preserved the globe in all $\mathbf{1 0}$ cases, and the pre-operative visual acuity was maintained or improved in all but 1 case. Six patients achieved a visual acuity of $6 / 60$ or better. Concomitant systemic immunosuppression was used in 6 cases and a subsequent penetrating keratoplasty performed in 3 cases. Lamellar keratoplasty provides a valuable method of preserving the integrity of the globe and maintaining useful vision in these difficult cases.
\end{abstract}

The major indications for lamellar keratoplasty are either optical, ${ }^{1}$ therapeutic ${ }^{2}$ or tectonic in nature. The value of a tectonic lamellar keratoplasty in preserving the integrity of the globe was demonstrated by Paufique in $1950,{ }^{3,4}$ but in recent years the popularity of this technique has diminished. ${ }^{5}$ This decline in popularity may be due to several factors. Lamellar keratoplasty is viewed as a technically more difficult procedure than penetrating keratoplasty. The results obtained with penetrating keratoplasty have

Correspondence to: D. A. R. Bessant, Moorfields Eye Hospital, City Road, London EC1V 2PD, UK.

Eye (1994) 8, 22-28 C 1994 Royal College of Ophthalmologists continued to improve and the visual outcome of a penetrating graft may be superior. ${ }^{6}$ Despite this, lamellar keratoplasty retains several advantages over a penetrating graft, not least the greatly reduced risk of injection. We feel that recent advances in microsurgical techniques made possible by the advent of diamond knives, viscoelastic agents and cyanoacrylate adhesives have improved both the technique and the results obtained from lamellar keratoplasty. ${ }^{7.8}$

Corneal ulceration and perforation may occur in the course of a number of local and systemic autoimmune diseases, including Mooren's ulceration, rheumatoid arthritis, Sjögren's syndrome, Wegener's granulomatosis, polyarteritis nodosa, systemic sclerosis and StevensJohnson syndrome..$^{.16}$ Ulceration may occur acutely during an active phase of the autoimmune process or it may commence later as a result of the hostile ocular surface environment, including factors such as conjunctival cicatrisation, lid distortion, trichiasis, entropion and keratoconjunctivitis sicca, that has been produced by earlier disease activity. It is important to make this distinction in each clinical case because the pathogenesis of the ulcer will determine the need for systemic immunosuppression and measures to control ocular surface disease. Ulceration may threaten vision by encroaching on the visual axis, by producing astigmatism, and ultimately by perforation of the globe. In the active phase of an autoimmune disease conjunctival recession and systemic immunosuppression may prevent progression of an ulcer, ${ }^{17-20}$ but sometimes, possibly due to the delay in the onset of effect of such drugs, perforation occurs in spite of these measures and keratoplasty is required to maintain the integrity of the globe.

In this paper we review a series of 10 patients in whom a tectonic graft was indicated for perforation or impending perforation. We aim to demonstrate that the modern microsurgical technique for lamellar keratoplasty can be 
versatile enough to become the technique of choice for restoring the integrity of the globe when corneal ulceration and perforation occur in the course of one of the aforementioned inflammatory diseases.

\section{PATIENTS AND METHODS}

The records of ten consecutive patients operated on by, or under the direct supervision of, a single surgeon (J.K.G.D.) between 1988 and 1992 were analysed retrospectively. Keratoplasty was performed when the integrity of the eye was threatened by perforation or impending perforation and when conservative measures such as the use of a soft contact lens and cyanoacrylate glue had failed to stabilise the situation. Patients were started on systemic immunosuppression prior to surgery when there was clinical evidence of active systemic or ocular inflammation. Oral prednisolone was prescribed in 6 cases and cyclophosphamide in 1 case. In 1 patient with relentlessly progressive Mooren's ulceration cyclosporin was combined with prednisolone. Immunosuppressive therapy was monitored with regular full blood counts. Lamellar grafts were of a number of different sizes and shapes according to the dimensions and location of the ulcer, and included horseshoe-shaped grafts for peripheral ulceration. General anaesthesia was employed unless felt to be contraindicated. The surgical technique is illustrated in Figs. 1-4.

The recipient cornea was dissected first. The extent and depth of the dissection was designed to remove all affected corneal tissue whilst preserving as much healthy host tissue as possible. Dissection of the recipient was greatly facilitated in those cases where perforation had occurred by re-forming the anterior chamber at the outset. If the perforation had not previously been successfully closed with cyanoacrylate adhesive then this procedure was performed before initiating the dissection. We utilised a gluing technique similar to that described by Hyndiuk and co-workers. ${ }^{21}$ A 2-3 mm diameter circle was punched using a skin biopsy punch from a sheet of plastic drape material. The circle of drape material was then attached to the end of a wooden 'orange-stick' using ophthalmic ointment or lubricating jelly. A drop of adhesive was placed on the other side of the drape material and then pressed gently onto the site of the perforation for a minute to allow it to set. Further patches were then applied as required. The viscoelastic agent sodium hyaluronidate (Healon, Kabi Pharmacia) was then introduced through a paracentesis to maintain both the anterior chamber depth and the intraocular pressure of the host eye.

The limits of the area to be resected were defined with a circular trephine where possible. In cases where this would have resulted in the removal of too much normal host tissue a freehand dissection was used instead and a horseshoe-shaped graft performed. In these cases a template which exactly matched the area to be resected was cut from plastic surgical drape material whilst the host globe was still fully formed. A diamond knife was used to make a superficial incision around the area to be resected.
This incision was then deepened to the required depth in one quadrant of the cornea or sclera to permit a lamellar dissector to be introduced parallel to the surface of the cornea. These steps may be facilitated by using an adjustable micrometer or stepped diamond knife to ensure that the incisions are made to the requisite depth. Lamellar dissection was then carried out, undermining the edges at the margin of the resected tissue, and approaching the thinnest or perforated area last. When the dissection was complete the lamellar flap was grasped with forceps, carefully peeled back and removed from the eye.

A whole donor eye was always used to provide the graft and dissection was carried out by a 'closed' technique. The donor eye was securely mounted in a Micra eyestand to maintain intraocular pressure and a small incision made to an appropriate depth close to the limbus with a diamond knife. The graft material was cut so that its thickness exceeded the depth of the recipient bed. A blunt lamellar dissector was introduced through this incision and a plane of cleavage obtained. A lamellar graft of an appropriate size was dissected by moving the spatula gently from side to side within the cleavage plane. It is important to maintain a uniform thickness in the graft so that vertical pressure is avoided during dissection. When the dissection had been completed a trephine of suitable diameter was used to cut the graft where possible. A freehand technique utilising the previously prepared plastic drape template was employed for unusually shaped grafts. Where the recipient site included the limbus a corneoscleral donor button was utilised.

The grafts were sutured into the lamellar bed with interrupted 10-0 Nylon sutures on the corneal aspect and 9-0 Nylon on the scleral side where applicable. Subconjunctival antibiotics (genticin $20 \mathrm{mg}$ or cefuroxime $125 \mathrm{mg}$ ) and betamethasone $(5 \mathrm{mg})$ were used. Post-operatively patients received topical steroid (dexamethasone $0.1 \%$ ) and antibiotic (chloramphenicol $0.5 \%$ ) therapy for a minimum of 6-8 weeks.

\section{RESULTS}

Details of the 10 patients studied together with a summary of their management appear in Table I. Five patients had at the time of presentation already been diagnosed as having rheumatoid arthritis, 2 had previously suffered an acute episode of erythema multiforme major (Stevens-Johnson syndrome), and 3 were given the diagnosis of Mooren's ulceration based on clinical features typical of this condition in the absence of any demonstrable systemic disease. Perforation had occurred in 8 of the 10 affected eyes of these patients prior to their first lamellar keratoplasty.

In all 10 cases lamellar keratoplasty restored the integrity of the globe and no eye was lost during the period of follow-up. Following the initial lamellar keratoplasty 2 patients underwent repeated lamellar grafting due to progression of the underlying disease process (cases 3 and 10). Case 10, in which two further grafts were performed due to recurrent disease activity, is described in detail 

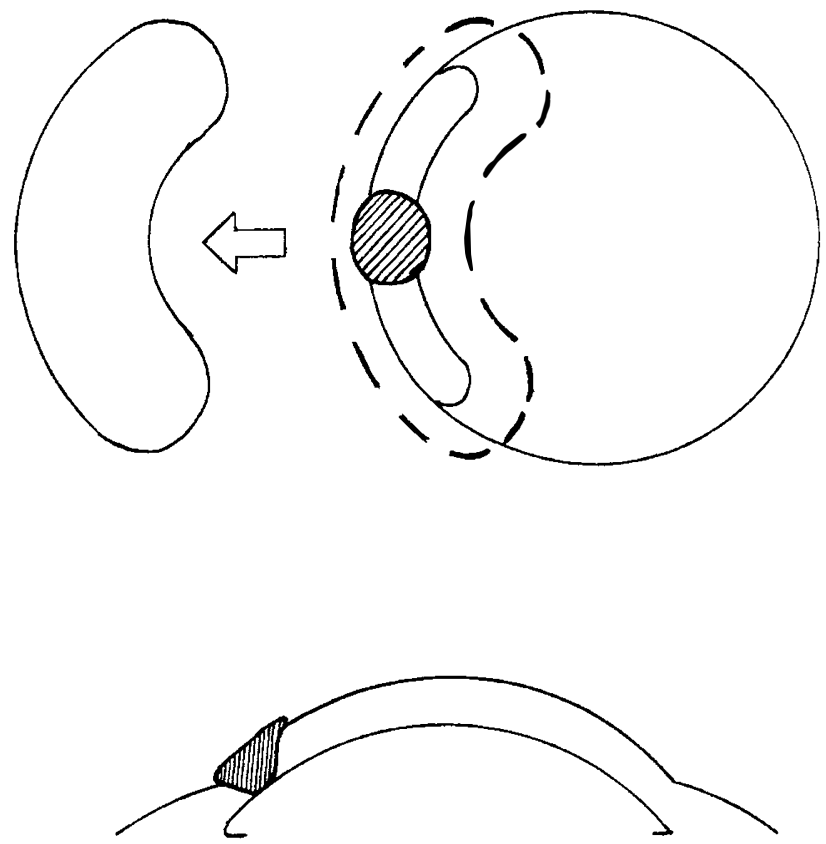

Fig. 1. The perforation site in a peripheral corneal ulcer is sealed with cyanoacrylate adhesive. Healon is then introduced into the anterior chamber via a paracentesis to re-form it prior to lamellar dissection. The area to be resected is defined and a template of this area cut from plastic surgical drape material whilst the host globe is still fully formed.
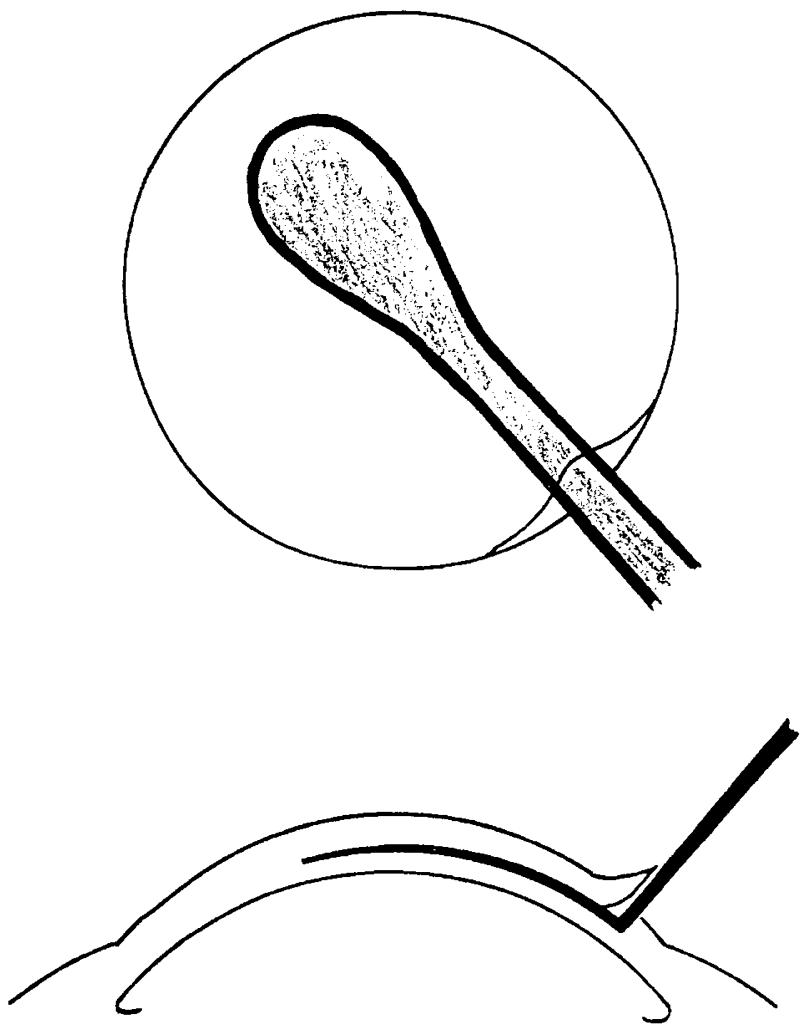

Fig. 3. With a whole donor eye securely mounted in a Micra eyestand a small incision is made to an appropriate depth close to the limbus. A blunt lamellar dissector is introduced and moved from side to side within the cleavage plane until a sufficient area has been dissected. It is important to maintain a uniform thickness in the graft so that vertical pressure is avoided during dissection.
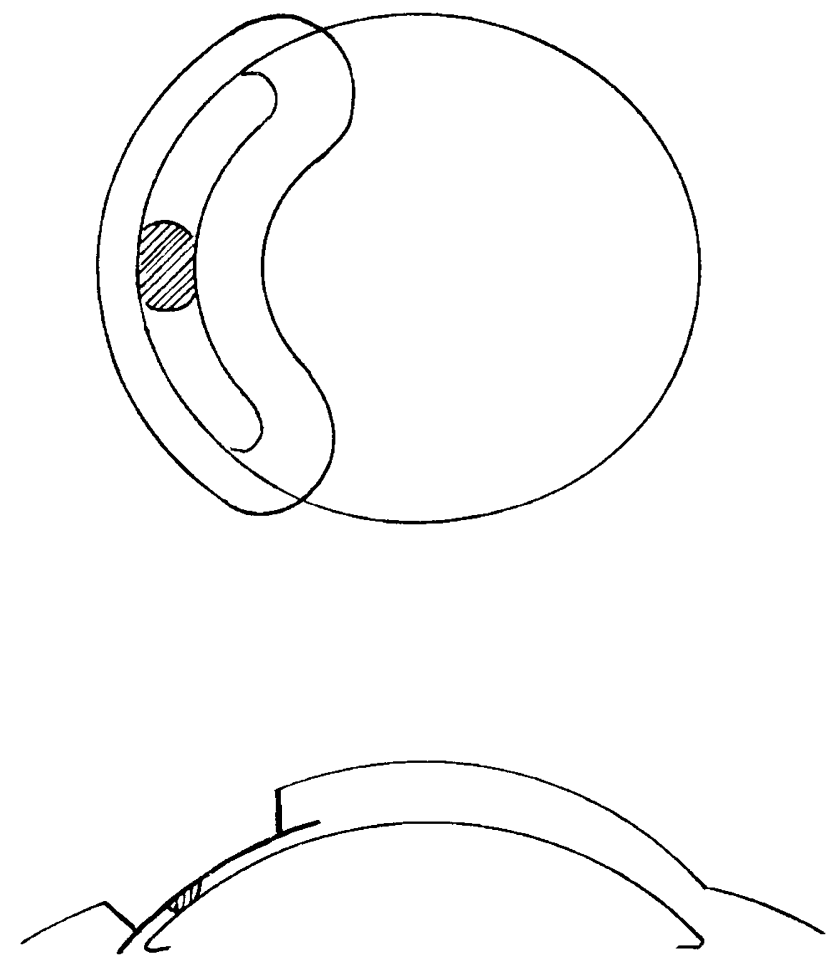

Fig. 2. A stepped diamond knife is used to make a superficial incision around the defined area. In one quadrant this incision is deepened to allow' a lamellar dissector to be introduced parallel to the surface of the cornea. Dissection is carried out, undermining the edges at the margin of the resected tissue, and approaching the thinnest area last. The flap is then peeled back and removed.
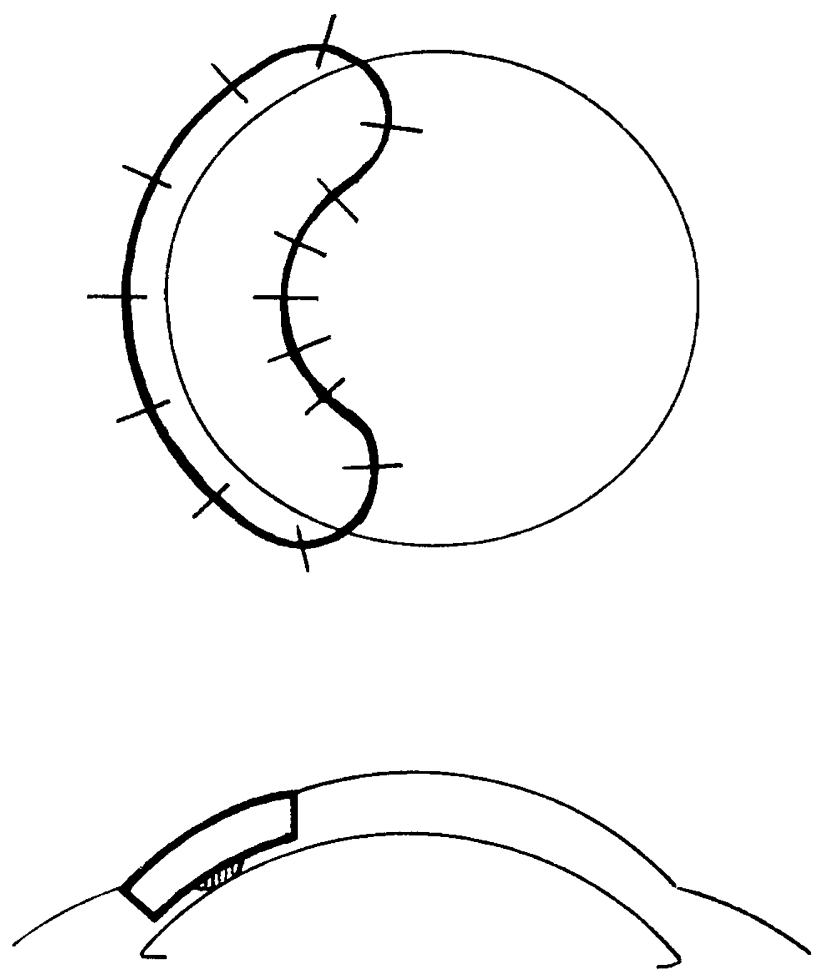

Fig. 4. The donor button is cut by a freehand technique which utilises the previously prepared template (for those cases requiring circular grafts a trephine is used). For a graft involving the limbus a corneoscleral donor button is used.The graft is sutured in place with interrupted 10-0 Nylon sutures on the corneal aspect and 9-0 Nylon on the scleral side respectively. 
Table I. Patient information

\begin{tabular}{|c|c|c|c|c|c|c|c|}
\hline \multirow{2}{*}{$\frac{\text { Case }^{\mathrm{a}}}{1(80 \mathrm{~F})}$} & \multirow{2}{*}{$\frac{\text { Diagnosis }}{\text { RA }}$} & \multirow{2}{*}{$\frac{\text { Perforated }}{\text { Yes }}$} & \multirow{2}{*}{$\begin{array}{l}\text { Surgery } \\
\text { OS, LK }\end{array}$} & \multicolumn{2}{|c|}{ VA (pre/post-op.) } & \multirow{2}{*}{$\begin{array}{l}\text { Notes } \\
\text { Pred. }\end{array}$} & \multirow{2}{*}{$\frac{\text { Follow-up (months) }}{15}$} \\
\hline & & & & $\mathrm{HM}$ & $\mathrm{CF}$ & & \\
\hline $2(49 M)$ & Mooren`s & Yes & OD, LK & $6 / 24$ & $6 / 12$ & & 12 \\
\hline $3(74 \mathrm{~F})$ & RA & No & $\mathrm{OD}, \mathrm{LK}(2)$ & $\mathrm{CF}$ & $6 / 36$ & Pred., PK & 26 \\
\hline $4(48 \mathrm{~F})$ & RA & Yes & OS, LK & $6 / 38$ & $6 / 18$ & Pred. & 10 \\
\hline $5(40 F)$ & SJS & Yes & $\mathrm{OD}, \mathrm{LK}$ & $\mathrm{PL}$ & $\mathrm{CF}$ & & 3 \\
\hline $6(54 \mathrm{~F})$ & RA & Yes & OD, LK & $\mathrm{CF}$ & $6 / 24$ & & 19 \\
\hline $7(78 \mathrm{~F})$ & RA & Yes & OS, LK & $\mathrm{CF}$ & $\mathrm{CF}$ & Pred/CPM & 1 \\
\hline $8(18 \mathrm{M})$ & Mooren's & Yes & OD. LK & $6 / 60$ & $6 / 60$ & Pred., PK & 7 \\
\hline $9(44 \mathrm{~F})$ & SJS & Yes & OS, LK & $\mathrm{HM}$ & $\mathrm{CF}$ & $\mathrm{PK}(2)$ & 14 \\
\hline $10(49 \mathrm{M})$ & Mooren`s & No & OS, LK(3) & $6 / 6$ & $6 / 18$ & Pred/CYC & 26 \\
\hline
\end{tabular}

RA, rheumatoid arthritis: SJS, Stevens-Johnson synrome; OD, right eye; OS, left eye; LK, lamellar keratoplasty: VA, visual acuity: HM. hand movements; CF; counting fingers; PK, penetrating keratoplasty; Pred., prednisolone; CPM, cyclophosphamide; CYC, cyclosporin.

'Information in parentheses is the patient's age (in years) and sex.

below. Case 3 was also one of the group of 3 patients who went on to have a penetrating keratoplasty. One patient in this group required a penetrating graft in order to ensure the complete removal of potentially infected tissue after an infiltrate developed in tissue adjacent to the lamellar graft and then failed to respond to adequate antimicrobial therapy (case 8). Visual rehabilitation was the main indication for grafting in the other two cases (cases 3 and 9).

The best -corrected pre-operative visual acuity was always assessed after the application of glue and the fitting of bandage contact lenses where appropriate, to reflect the real visual potential of the eye. The visual acuity recorded from perforated eyes with flat anterior chambers prior to gluing was frequently much worse. Post-operative visual acuities were taken from the record of the most recent outpatient appointment. This clearly reflects the result of further intervention in some cases, but may be considered a more realistic appraisal of the longer term visual prognosis for eyes undergoing lamellar grafting in these circumstances. The pre-operative visual acuity was maintained or improved in 9 of these 10 cases. Six of these had a final visual acuity of $6 / 60$ or better, whilst the remaining 4 retained Counting Fingers vision. In the patient in whom visual acuity deteriorated this was due to relentless progression of the underlying disease process and the need for several further surgical procedures.

The follow-up time for patients in this study varied from 1 to 26 months. The shorter periods of follow-up were all attributable to patients being returned to the care of the ophthalmic surgeon who had originally referred them to Moorfields Eye Hospital.

\section{CASE HISTORIES}

\section{Case 4 (Rheumatoid Arthritis)}

A 48-year-old woman was initially referred for management of a perforated left corneal ulcer. She had been diagnosed 2 years previously as having rheumatoid arthritis and had a 12 month history of 'dry eyes'. She had originally been treated at the referring hospital with guttae genticin hourly for a left central corneal ulcer from which Micrococcus and Acinetobacter had been cultured. After the microbial keratitis had settled a persistent epithelial defect had remained which progressed to become a perforation.

On presentation her corrected visual acuities were 6/9 right and 6/36 left. The left cornea was thinned centrally with a perforation and the anterior chamber was flat. The right cornea and conjunctiva were covered by punctate epithelial erosions. The marginal tear film was very thin with a rapid tear break-up time in both eyes. A silicone rubber bandage contact lens was fitted in the left eye. Both eyes were treated with preservative-free guttae hypromellose hourly and the left eye received unpreserved guttae chloramphenicol four times daily. Occlusive cautery to all four lacrimal punctae was carried out to prevent tear drainage.

Two days later the anterior chamber remained very shallow and the corneal perforation was sealed with cyanoacrylate adhesive and the bandage contact lens reinserted. Following this procedure the anterior chamber deepened and a moderate uveitis with plasmoid aqueous and a fibrin clot over the pupil was noted. As well as topical guttae dexamethasone 2 hourly to the left eye she was given a single intravenous dose of methylprednisolone $500 \mathrm{mg}$ and cyclophosphamide $500 \mathrm{mg}$. This dose was repeated 3 days later and, because this appeared to produce a dramatic improvement, she was started on prednisolone $40 \mathrm{mg}$ and cyclophosphamide $150 \mathrm{mg}$ per day orally. Her cyclophosphamide was stopped 6 weeks later due to a low lymphocyte count and the dose of prednisolone gradually reduced to $15 \mathrm{mg}$ once a day.

Ten weeks following her initial admission she was readmitted with a recurrent left corneal perforation at the original site (the bandage contact lens having been removed at clinic 16 days previously). Left visual acuity was Counting Fingers and the anterior chamber was flat. A lamellar keratoplasty was performed. The anterior chamber was re-formed with Healon via a paracentesis prior to dissection of the recipient bed. A $5.1 \mathrm{~mm}$ trephine was used to prepare both the host and the donor lamellar tissue and the graft was secured with 12 interrupted $10-0$ Nylon sutures. At follow-up 8 months later her left visual acuity was $6 / 18$.

\section{Case 5 (Stevens-Johnson Syndrome)}

A 40-year-old female black Jamaican had suffered eryth- 
ema multiforme major 10-years earlier. Her right eye had been lost following a corneal perforation. She was referred from Jamaica for management of a central corneal perforation in her remaining left eye. This had followed frequent recurrent episodes of corneal ulceration which had required weekly hospital visits for 3 years.

The right eye was phthisical. The left eye had a central corneal perforation within a central zone of thinning with scarring, ulceration and a minimal focal corneal infiltrate. The cornea was deeply vascularised in all quadrants, there was a flat anterior chamber and a mature cataract. There was moderate conjunctival shortening in all fornices, a very poor tear film and lacrimal punctae occluded by scarring. Corneal cultures were negative but the patient was treated with intensive topical and systemic antibiotics for presumed microbial keratitis for 3 days. The anterior chamber could not be re-formed fully with a therapeutic contact lens. The patient underwent a cataract extraction, without a lens implant, through a peripheral corneal section followed by a tectonic lamellar keratoplasty. At the completion of this procedure an inferior conjunctival bucket-handle flap was elevated and sutured into the superior limbus after the removal of all host and donor epithelium.

Three months later the patient had a visual acuity of Counting Fingers, a full field to confrontation, and independent navigating vision. The intraocular pressure was normal, the conjunctival flap stable and there had been no further episodes of corneal ulceration. The combination of a lamellar keratoplasty with a conjunctival flap provided a stable ocular surface epithelium resistant to ulceration in a dry eye with poor potential for corneal re-epithelisation.

\section{Case 10 (Mooren's Ulceration)}

This 49-year-old diabetic man was referred with a 6 week history of pain and irritation in his left eye. On presentation his visual acuity ws $6 / 5$ in each eye. He was noted to have a peripheral corneal ulcer affecting the superotemporal limbus and extending for three clock-hours. The corneal thickness was reduced by half in the region of this gutter. History taking, clinical examination and a full immunological screen failed to reveal any evidence of an underlying systemic disease, and therefore a diagnosis of Mooren's ulceration was made.

The patient was initially treated with topical steroids and antibiotics and a therapeutic contact lens. He continued, however, to have a number of exacerbations of his condition and despite the introduction of systemic immunosuppression (prednisolone $80 \mathrm{mg} /$ day and cyclosporin $150 \mathrm{mg}$ twice daily) and a local conjunctival recession performed 2 weeks after presentation his ulcer progressed until it involved half the corneal limbus. Conservative measures were maintained for 9 months until the left cornea was judged to be in imminent danger of perforation.

A horseshoe-shaped lamellar corneoscleral graft was performed at the superior limbus using the technique described earlier. Two weeks following this procedure the patient's visual acuity was $6 / 6$. The eye remained stable for 6 months until a reactivation of the melting process resulted in a tiny corneal perforation at the superotemporal limbus. This was initially treated successfully with cyanoacrylate adhesive and a bandage contact lens, but 6 weeks later the perforation enlarged and a substantial iris prolapse occurred. A second horseshoe-shaped lamellar keratoplasty was performed over the site of the first graft and encompassing six clock-hours of the superotemporal limbus.

Ten months later recurrent disease activity led to marked thinning of the stroma of the donor tissue and a further lamellar graft was carried out. Subsequent management has included continuing systemic immunosuppression and two further conjunctival recessions. The patient's current visual acuity is $6 / 18$.

\section{DISCUSSION}

Lamellar keratoplasty forms part of a combined medical and surgical approach to the management of patients who suffer corneal ulceration and perforation secondary to local or systemic inflammatory diseases. In some of these disorders, such as Mooren's ulceration and Wegener's granulomatosis, peripheral corneal ulceration appears to be the direct result of the destruction of corneoscleral tissue by the underlying autoimmune reaction, possibly mediated in part by an occlusive vasculitis of the anterior ciliary vessels. ${ }^{10.12}$ In patients in whom the autoimmune process is active, corneal ulceration may respond to conjunctival recession and systemic immunosuppression before perforation occurs, so that tectonic keratoplasty becomes unnecessary. Corneal ulceration in these conditions may, however, progress very rapidly and come to involve a great deal of the limbus, and since it may take immunosuppressive agents up to 8 weeks to bring the inflammatory process under control there is a high risk of perforation. ${ }^{19.20}$

At the other end of the spectrum of inflammatory conditions Stevens-Johnson syndrome predisposes to corneal ulceration by producing a hostile ocular surface environment. The acute episode of haemorrhagic conjunctivitis gives rise to extensive conjunctival cicatrisation which in turn may lead to symblepharon, trichiasis, entropion, and a deficiency of both the aqueous and mucinous phases of the tear film. It is this hostile environment rather than the original autoimmune process which is responsible for corneal ulceration and perforation in these patients. Adequate management of these cases requires careful identification of factors which may lead to ulceration and the appropriate use of preservative-free topical lubricants, punctal occlusion, lid surgery, cryotherapy or electrolysis for trichiasis, and if necessary therapeutic contact lenses. ${ }^{22}$ In some cases limbal autografting may also be used to provide the cornea with a stable epithelium.

The pathogenesis of corneal ulceration in rheumatoid arthritis may either be inflammatory or involve the associated keratoconjunctivitis sicca. In each case the clinical features, such as the presence or absence of scleritis adjacent to the area of ulceration and the severity of the sicca 
syndrome, together with careful observation of the response to initial therapy will help to identify the factors responsible for a particular episode of ulceration. Further management, which may involve the use of oral immunosuppression, can then be planned. In our series 4 of the 5 patients with rheumatoid arthritis were started on oral prednisolone prior to grafting and in 1 case this was combined with cyclophosphamide. This does not indicate that immunosuppression is required for the majority of cases of rheumatoid corneal ulceration since the patients in this series are those whose ulceration could not be halted by conservative measures. Ulceration due to inadequate tear production can frequently be managed without resort to surgery.

Some authors have stressed the need for immunosuppression in all cases of inflammatory peripheral ulcerative keratitis undergoing keratoplasty, indicating that recurrent inflammation may otherwise lead to graft failure. ${ }^{23}$ In our experience this is not always the case and we feel that it is important to identify those patients in whom inflammation is active or likely to recur so as to avoid unnecessary immunosuppression. Clearly in patients with StevensJohnson syndrome and in some of those with rheumatoid arthritis these agents would be ineffective and potentially dangerous.

When perforation does occur or is imminent, lamellar keratoplasty offers several advantages over a penetrating graft in these patients. There is a high risk of rejection of a peripheral penetrating graft in an already inflamed eye. The location of the graft places donor antigens immediately adjacent to the vascular tissue of the corneal limbus, stimulating the afferent limb of the host immune response, whilst pre-existing inflammation stimulates the efferent limb of this response. The risk of rejection is greatly reduced for a lamellar graft and if rejection does occur it is easy to control. If perforation has not yet occurred a lamellar graft is not an intraocular procedure and it therefore allows the surgeon to avoid the complications, such as cataract and endophthalmitis, associated with entering the anterior chamber in the process of a penetrating graft. A lamellar keratoplasty permits the preservation of the maximum amount of host tissue and adds to the thickness of the cornea. This may reduce the risk of recurrent perforation if the corneal melting process reactivates, as it may well do in conditions such as Mooren's ulceration.

Lamellar keratoplasty is contraindicated in the presence of overt infection. One patient in this series underwent a penetrating keratoplasy subsequent to the initial lamellar graft specifically to remove tissue adjacent to the graft that appeared to be infected. There was no sign of infection at the time of surgery, but a dense corneal infiltrate appeared 6 months after the initial graft.

The optical results of lamellar keratoplasty may be impaired both by any irregularity of the interface between host and donor tissue and by lipid deposition in this interface. This is a significant disadvantage compared with a penetrating keratoplasty when a graft involving the visual axis is planned because it has been shown to result in a reduced post-operative visual acuity. ${ }^{6}$ However, many inflammatory diseases tend to produce peripheral ulceration which can be adequately controlled with a horseshoe-shaped lamellar graft not involving the visual axis.

With the advent of modern microsurgical techniques we feel that a lamellar keratoplasty is now technically as simple to perform as a penetrating graft. Tissue adhesives and viscoelastic agents allow the intraocular pressure and anterior chamber depth of the perforated host eye to be stabilised prior to dissection of the lamellar bed. Ultrasharp and ultrathin micrometer diamond knives with preset steps permit the depth of dissection to be accurately determined. In 1980 Taylor and Stern performed lamellar keratoplasty on only 15 of 50 eyes of patients with corneal ulceration and perforation..$^{24}$ In 1991, however, Raizman and co-workers ${ }^{24}$ stated that in their series of 17 patients with inflammatory peripheral ulcerative keratitis 'lamellar keratoplasty was performed when possible' (13 cases), and that penetrating keratoplasty was 'reserved for eyes with the most extensive necrosis'. The increased use of lamellar grafts by the second group of authors may at least in part be the result of recent advances in surgical technique.

In summary we feel that lamellar keratoplasty has a valuable role to play in the management of inflammatory diseases leading to corneal ulceration and perforation. It is particularly suitable for peripheral perforations. In the presence of active corneal infection a penetrating graft should be used. A lamellar keratoplasty may stabilise a difficult situation following a perforation and permit an eye to be salvaged that would otherwise be lost. Later, when the eye is less inflamed, a penetrating graft can be performed with a much reduced risk of rejection if it is required for visual rehabilitation.

Key words: Cornea, keratoplasty, Lamella, Mooren's ulceration, Perforation, Rheumatoid arthritis, Stevens-Johnson syndrome.

\section{REFERENCES}

1. Paufique L, Charleux J. Lamellar keratoplasty. In: Casey TA, editor. Corneal grafting. London: Butterworth, 1972: 121-76.

2. Pearlman G, Susal AL, Hulshaw J, et al. Recurrent pterygium and treatment with lamellar keratoplasty with presentation of a technique to limit recurrences. Ann Ophthalmol 1970;2:763-71.

3. Paufique L. Indications for the therapeutic lamellar corneal graft. Am J Ophthalmol 1950;33:24-5.

4. Paufique L, Philps S. Acute eye lesions treated by lamellar corneal grafting. Br J Ophthalmol 1950;34:746-8.

5. Arentsen JJ, Morgan B, Green WR. Changing indications for keratoplasty. Am J Ophthalmol 1976;81:313-8.

6. Richard JM, Paton D, Gasset AR. A comparison of penetrating and lamellar keratoplasty in the surgical management of keratoconus. Am J Ophthalmol 1978;86:807-11.

7. Hirst LW, De Juan E Jr. Sodium hyaluronidate and tissue adhesives in treating corneal perforations. Ophthalmology 1982;89:1250-3.

8. Maguen E, Nesburn AB, Macy JI. Combined use of sodium hyaluronate and tissue adhesive in penetrating keratoplasty of corneal perforation. Ophthalmic Surg 1984;15-55.

9. Wood TO, Kaufman HE. Mooren's ulcer. Am J Ophthalmol 1971;71:417-21. 
10. Jayson MIV, Easty DL. Ullceration of the cornea in rheumatoid arthritis. Ann Rheum Dis 1977;36:428.

11. Sevel D. Necrogranulomatous keratitis associated with Wegener's granulomatosis and rheumatoid arthritis. Am J Ophthalmol 1967;63:250-5.

12. Krachmer JH, Laibson RR. Corneal thinning and perforation in Sjögren's syndrome. Am J Ophthalmol 1974;78: 917-20.

13. Austin P, Green WR, Sawyer DC, et al. Peripheral corneal degeneration and occlusive vasculitis in Wegener's granulomatosis. Am J Ophthalmol 1978;85:311-7.

14. Moore JG, Sevel D. Corneoscleral ulceration in polyarteritis nodosa. Br J Ophthalmol 1966;50:651-5.

15. Horie K, Nishi M, Sawa M, Mochizuki M. A case of peripheral corneal ulcer accompanied by progressive systemic sclerosis. Nippon-ganka-Gakkai-Zashi 1992;96:922-9.

16. Aristikatis MJ. Ocular aftermath of Stevens-Johnson syndrome. Arch Ophthalmol 1973;90:376-9.

17. Brown SI. Mooren's ulcer: treatment by conjunctival excision. Br J Ophthalmol 1975;59:675-82.
18. Wilson FM, Grayson M, Ellis FD. Treatment of corneal ulcers by limbal conjunctivectomy. Br J Ophthalmol 1976; 60:713-9.

19. Foster CS. Immunosuppressive therapy for external ocular inflammatory disease. Ophthalmology 1980;87:140-50.

20. Tauber J, Sainz de la Maza M, Hoang-Xuan T, Foster CS. An analysis of decision making regarding immunosuppressive chemotherapy for peripheral ulcerative keratitis. Cornea 1990;9:66-73.

21. Hyndiuk RA, Hull DS, Kinyoun JL. Free tissue patch and cyano-acrylate adhesive in corneal perforation. Ophthalmic Surg 1974;5:50.

22. Leibowitz HM, Rosenthal P. Hydrophilic contact lenses in corneal disease. Arch Ophthalmol 1971;85:163-6.

23. Raizman MB, Sainz de la Maza M, Foster CS. Tectonic keratoplasty for peripheral ulcerative keratitis. Cornea 1991;10: 312-6.

24. Taylor DM, Stern AL. Reconstructive keratoplasty in the management of conditions leading to corneal destruction. Ophthalmology 1980;87:892-904. 